\title{
Optimal scheduling of low-dose metronomic chemotherapy: an in-silico analysis
}

\author{
Bálint Péceli, Dániel András Drexler and Levente Kovács
}

\begin{abstract}
Low-dose metronomic (LDM) chemotherapy shows promising results in cancer treatment. However, the scheduling of the therapy, including the determination of the optimal biologic dose is usually based on empiricism. This paper contributes to an in-silico analysis targeting parameter optimization of LDM chemotherapy design. The in-treatment tumor growth model used by the analysis formulates tumor proliferation and necrosis, dead tumor cell washout, as well as pharmacokinetics and pharmacodynamics of the administered drug. The model parameters are identified based on mouse experiments. The optimization goal is the minimization of the total amount of drug delivered throughout the full length of the therapy with governing constraints ensuring the efficacy of the treatment. Results show that a clear optimum exists in the scheduling of the treatments, that is, an optimal choice for the rest periods can be done. The optimum is independent of the length of the therapy, and only slightly depends on the parameter sets of the individual patients.
\end{abstract}

\section{INTRODUCTION}

Low-Dose Metronomic chemotherapy (LDM) was first proposed 20 years ago as a therapy with low dose high density drug administration versus Maximum Tolerated Dose (MTD) treatment [1]. Initially, it was considered as an antiangiogenic therapy inhibiting the formulation of blood vessels supporting the growth of the tumor cells. Since then, LDM strategies turned out to be effective also in immunotherapy and its direct effects on cancer cells has been discovered as well. It has been examined as a monotherapy and in combination with other treatment methods like MTD chemotherapy and radiotherapy. Recent results show that LDM strategies can outperform MTD strategies, especially for fast-growing tumors [2]. Reviews on the study of LDM therapy can be found in Loven et al. [3] and André et al. [4].

From engineering perspective, LDM can be modelled as a discrete time control problem with impulsive inputs. The theory of impulsive control is well known from e.g [5] and [6]. The main concept is that inputs are modelled as instant changes in the values of some state variables, not

This project has received funding from the European Research Council (ERC) under the European Union's Horizon 2020 research and innovation programme (grant agreement No 679681).

Project no. 2019-1.3.1-KK-2019-00007. has been implemented with the support provided from the National Research, Development and Innovation Fund of Hungary, financed under the 2019-1.3.1-KK funding scheme.

The present work has also been supported by the Hungarian National Research, Development and Innovation Office (2018-2.1.11-TÉT-SI-201800007 and SNN 125739).

The Authors are with the Physiological Controls Research Center within the Research and Innovation Center of Óbuda University, Budapest, Hungary pecbalegmail.com, drexler.danielenik.uni-obuda.hu,

kovacs@uni-obuda.hu as explicit input signals as in traditional control theory. We started to adapt this technology to cancer treatment a few years ago, see [7] and [8]. Research studies targeting similar goals but using different models can be found in [9] and [10].

The objective of this paper is to propose optimal schedule of LDM chemotherapy. This is done by presenting a detailed in-silico analysis of our therapy generation algorithm using an impulsive discrete time patient model, introduced in [7] and [8]. Our main findings are that an optimal choice for rest periods between treatments can be done, this choice is independent from the length of the therapy and correlates with the clearance and median effective dose of the applied chemotherapeutic drug (pegylated liposomal doxorubicin) for the individual patients. The rest of the paper is structured as follows: Chapter II describes the tumor growth model as the evolution of previous studies. Chapter III expounds the therapy generation algorithm. Chapter IV presents the tools and methods used for the analysis of which results are discussed in Chapter V. Finally, Chapter VI concludes the paper.

\section{THE TUMOR GROWTH MODEL}

The tumor growth model used in this paper is based on a minimalistic approach (i.e., using as few state variables and parameters as possible), which was refined in several iterations after its first publication. The final model is presented here as the result of a step-by-step development process.

The initial model [11] describes tumor cell proliferation and the pharmacokinetics of the applied angiogenic inhibitor using only two state variables: the tumor volume and the inhibitor serum level. This model is extended in [12] by incorporating the effects of necrosis and the pharmacodynamics of the drug. Tumor volume is divided here to living and dead tumor cells, so the number of state parameters is increased by one. In [13], [14], the direction of the research changes from angiogenic inhibition to chemotherapy. According to this change, the model is modified by incorporating the washout of dead tumor cells, which is a critically important phenomena during chemotherapy. The so far final extension of the model presented in [8] increases the number of state variables by one again, refining the pharmacokinetics with the application of a two-compartment system (central and peripheral). This representation showed better results during parameter identification than the formal description with three sate variables. Furthermore, the inputs of the system are modelled as impulsive effects in the value of the state variable describing the drug level in the central 
compartment. This is in contrast with the classical approach, which formulates injections as direct, discrete-time inputs.

The tumor growth model used for the in-silico analysis is presented below in two forms. At first, an illustrative explanation is given using an analogy to formal reaction kinetics. Suppose that $X_{1}, X_{2}, X_{3}$ and $X_{4}$ are fictive species representing the volume of the living tumor cells, the volume of the dead tumor cells, the drug level in the central compartment and the drug level in the peripheral compartment, respectively. The tumor growth during chemotherapy is then given by the following reaction steps:

- $\mathrm{X}_{1} \stackrel{\mathrm{a}}{\longrightarrow} 2 \mathrm{X}_{1}$ : proliferation of living tumor cells;

- $\mathrm{X}_{1} \stackrel{\mathrm{n}}{\longrightarrow} \mathrm{X}_{2}$ : tumor cell necrosis;

- $\mathrm{X}_{2} \stackrel{\mathrm{w}}{\longrightarrow} \mathrm{O}$ : dead tumor cell washout;

- $\mathrm{X}_{1}+\mathrm{X}_{3} \stackrel{\mathrm{b}}{\longrightarrow} \mathrm{X}_{2}$ pharmacodynamics (the effect of the chemotherapeutic drug);

- $X_{3} \stackrel{c}{\longrightarrow}$ O: pharmacokinetics (depletion of the drug);

- $\mathrm{X}_{3} \underset{\mathrm{k}_{2}}{\stackrel{\mathrm{k}_{1}}{\rightleftarrows}} \mathrm{X}_{4}$ two-compartment pharmacokinetics (the spread of the drug in the body).

We give another representation of the tumor growth model, which uses system engineering terminology. The state-space model is given as follows:

$$
\begin{aligned}
& \dot{x}_{1}=(a-n) x_{1}-b \frac{x_{1} x_{3}}{E D_{50}+x_{3}} \\
& \dot{x}_{2}=n x_{1}+b \frac{x_{1} x_{3}}{E D_{50}+x_{3}}-w x_{2} \\
& \dot{x}_{3}=-\left(c+k_{1}\right) x_{3}+k_{2} x_{4}-b_{k} \frac{x_{1} x_{3}}{E D_{50}+x_{3}} \\
& \dot{x}_{4}=k_{1} x_{3}-k_{2} x_{4},
\end{aligned}
$$

in the intervals $\left[0, t_{1}\right],\left[t_{1}, t_{2}\right], \ldots,\left[t_{i}, t_{i+1}\right], \ldots$ for $i=1,2, \ldots$ The injections $u_{i}$ are administered in time instants $t_{i}, i=$ $1,2, \ldots$ and have effect on the third state as

$$
x_{3}\left(t_{i}^{+}\right)=x_{3}\left(t_{i}^{-}\right)+u_{i} .
$$

The state variables of the system are $x_{1}, x_{2}, x_{3}$ and $x_{4} . x_{1}$ is the time function of the proliferating tumor volume in $\mathrm{mm}^{3}, x_{2}$ is the time function of the dead tumor volume in $\mathrm{mm}^{3}, x_{3}$ is the time function of the drug level in the central compartment in $\mathrm{mg} / \mathrm{kg}$ and $x_{4}$ is the time function of the drug level in the peripheral compartment in $\mathrm{mg} / \mathrm{kg}$.

The output is the total tumor volume in $\mathrm{mm}^{3}$ that is the sum of the living and dead tumor cells:

$$
y=x_{1}+x_{2} .
$$

The dynamics of the total tumor volume is then described by the differential equation

$$
\dot{y}=a x_{1}-w x_{2},
$$

which means that the change of the measured tumor volume depends directly on the tumor growth rate constant $a$, the tumor washout rate constant $w$ and the actual volume of the proliferating and dead tumor cells.

The model parameters were identified based on real measurement data also in [8] based on the experiments described in [15]. The identified parameters for the seven mice not showing resistance against the administered drug in the experiments in [15] are presented in Table I. Note that the parameter $b_{k}$ on the right hand side of (3) was close to zero during the identification, therefore it is not present in the Table I and is neglected in our analysis.

\section{THERAPY DESIGN}

We use the tumor growth model described in Chapter II for developing optimal LDM chemotherapy. The related treatment scenario and the optimal control problem was founded in [16]. Here, the angiogenic inhibitor is administered to the patient in form of injections scheduled in fixed and equidistant time intervals called rest periods. Every time the doctor investigates the patient, the tumor volume is measured and recorded. In the next step, the doctor defines the desired volume of the tumor for the end of the following period. The optimal control problem is then to find the minimal amount of drug that is required to be administered to the patient such that the tumor volume at the end of the next period is less than or equal to the desired level.

A binary search algorithm was proposed to solve the optimal control problem [16], which was later modified and adapted to the new tumor growth model in [7]. We recall this procedure in Algorithm 1 due to its high importance with respect to our analysis.

The generation of the full therapy is constituted by running the search algorithm several times (defined by the total length of the therapy and the chosen length of the rest periods) consecutively, while giving the initial values of the state variables equal to their final values calculated by the previous execution of the algorithm. The very first initial value of the proliferating tumor volume $\left(x_{1}\right)$ is given by the identification process (see Table I), while the other three states (dead tumor volume $x_{2}$, drug level in the central compartment $x_{3}$ and drug level in the peripheral compartment $x_{4}$ ) are assumed to be zero for $k=0$. The reference volume is calculated in silico by giving $U_{M A X}$ dose of the drug, which is the MTD by default, but will be modified in the later analysis in Subsection V-C.

\section{ANALYSIS}

Given the therapy generation algorithm described in Chapter III, our aim was to find the optimal input parameter set, and particularly, the optimal schedule of injections in order to minimize the total amount of chemotherapeutic drug necessary for a successful therapy. Altogether, the input parameter set consists of the following constants:

- $U_{M A X}[\mathrm{mg} / \mathrm{kg}]$ : injection dose limit,

- $\epsilon$ : maximum tolerated distance from the desired tumor volume,

- TOL: maximum tolerated distance from the analytical optimum of the injection dose (also determining the stop condition of the algorithm),

- $L[$ days $]$ : total duration of the therapy, and

- $T_{s}[$ days $]$ : length of the rest periods. 
TABLE I

THE IDENTIFIED PARAMETERS FOR THE TUMOR GROWTH MODEL BASED ON MICE EXPERIMENTS [8]

\begin{tabular}{l|lllllll} 
Parameter & PLD2 & PLD3 & PLD4 & PLD5 & PLD6 & PLD9 \\
$a[1 /$ day $]$ & 0.110418406 & 0.215526754 & 0.16765648 & 0.140906773 & 0.146644234 & 0.10046402 & 0.06409301 \\
$b[1 /$ day $]$ & 0.420126468 & 0.471192264 & 0.769957493 & 0.414039983 & 0.397670985 & 0.638828893 & 0.228345556 \\
$c[1 /$ day $]$ & 0.920366148 & 0.711276923 & 1.123719728 & 0.591860057 & 0.635236124 & 0.745639795 & 1.217108287 \\
$E D_{50}[\mathrm{mg} / \mathrm{kg}]$ & 0.001479554 & $5.03 E-05$ & $8.96 E-05$ & 0.00072989 & 0.001534443 & 0.001392283 & 0.000323682 \\
$k_{1}[\mathrm{mg} / \mathrm{kg}]$ & 7.182480087 & 10.36900747 & 4.665498045 & 5.139064896 & 10.03181807 & 7.427887027 & 5.455678554 \\
$k_{2}[\mathrm{mg} / \mathrm{kg}]$ & 74.42509769 & 34.27463714 & 69.08963483 & 139.8784866 & 62.82274197 & 69.76133667 & 60.57456315 \\
$n[1 / \mathrm{day}]$ & 0.00026832 & 0.000269518 & 0.00032116 & 0.000277835 & 0.000276248 & 0.000344623 & 0.00028237 \\
$w[1 / \mathrm{day}]$ & 0.015339999 & 0.08779158 & 0.064449621 & 0.014813315 & 0.087397824 & 0.011934765 & 0.097799656 \\
$x_{1}(0)\left[\mathrm{mm}^{3}\right]$ & 14.05317925 & 130.6485947 & 45.75888757 & 15.44381186 & 24.07972372 & 22.39883988 & 95.50732075
\end{tabular}

Data: The initial values $x_{1}[k], x_{2}[k], x_{3}[k]$ and $x_{4}[k]$. The maximal drug injection $U_{M A X}$ and the accuracy parameters $\epsilon$ and $T O L$.

Result: The minimal drug dosage $u[k]$ that is required to reach the tumor volume in the vicinity of the desired tumor volume (parameterized by $\epsilon$ ) in the next step.

Let $u_{\max }=U_{M A X}$ and $u_{\min }=0$;

Let $u=U_{M A X}$. Calculate the tumor volumes in the next time instant $(k+1) T_{s}$ by solving the initial value problem on time interval $\left[k T_{s},(k+1) T_{s}\right]$ defined by (1)-(4) with initial values $x_{1}[k], x_{2}[k], x_{3}[k]+u, x_{4}[k]$, denote them by $x_{1}[k+1]$ and $x_{2}[k+1]$, and let the desired tumor volume in the next time instant be

$$
\begin{aligned}
& y_{\text {ref }}[k+1]:=x_{1}[k+1]+x_{2}[k+1] \text {; } \\
& \text { while } u_{\max }-u_{\min }>T O L \text { do } \\
& u=\left(u_{\max }-u_{\min }\right) / 2 \text {; } \\
& \text { Calculate the tumor volumes in the next time } \\
& \text { instant }(k+1) T_{s} \text { by solving the initial value } \\
& \text { problem on time interval }\left[k T_{s},(k+1) T_{s}\right] \\
& \text { defined by (1)-(4) with initial values } \\
& x_{1}[k], x_{2}[k], x_{3}[k]+u, x_{4}[k] \text {, denote them by } \\
& x_{1}[k+1] \text { and } x_{2}[k+1] \text {; } \\
& \text { Let } y[k+1]=x_{1}[k+1]+x_{2}[k+1] \text {; } \\
& \text { if } y[k+1]-y_{\text {ref }}[k+1]>\epsilon y_{\text {ref }}[k+1] \text { then } \\
& u_{\text {min }}:=u \\
& \text { else } \\
& u_{\max }:=u \\
& \text { end } \\
& \text { end }
\end{aligned}
$$

Algorithm 1: The search algorithm to find the minimal drug delivery for the next step [7]

Throughout the analysis we tuned the parameter $T_{s}$ defining the schedule of injections while keeping the remaining parameters fixed in several different configurations. The total amount of drug required to fulfill the generated therapy was calculated for each case and plotted against the length of the rest periods.

Although classical MTD (Maximum Tolerated Dose) therapies apply typically longer rest periods than ten days, our aim was to find the optimal schedule of LDM (Low-Dose Metronomic) precisely, instead of comparing the two types of therapy strategies. Therefore we focused only on intervals between 12 hours and ten days.

Note that we only investigated such configurations that resulted in successful therapies, i.e., where the tumor volume was reduced to zero or close to zero after the treatments. Therapy validation was carried out by comparing the series of desired tumor values with the output of the patient model under simulation (as defined by (6))

The drug was administered by means of injections distributed equidistantly in time for all the test cases. That means that the length of the rest periods $T_{s}$ was kept constant during the therapy. As the length of the therapy $L$ itself was also fixed, the number of injections was less for cases designed with longer rest periods than for cases with shorter ones. That might cause sudden jumps in the output curves if the maximal amount of drug administered via a single injection $U_{M A X}$ was limited. Moreover, the end of the therapy was defined as the last possible date of drug administration, and not as the target date of the full elimination of the tumor. Consequently, it might happen for some cases that the patient receives the final injection on the last days of the therapy, but the tumor will only be fully eliminated some days after.

We used the parameter set of patient PLD6 for therapy generation. This choice was made already in [7], since this patient had the largest $E D_{50}$ parameter, thus it requires the largest doses and serves as a worst case scenario. This way we had the possibility to compare the outputs with previous results.

As the therapy was generated every time for an individual patient, we further analyzed the algorithm to find inter-patient variability in relation with the optimal scheduling, and also to give a quantitative description of the correlations between the parameter sets of the patients and the generated therapies.

The patient model was implemented in Simulink and tested by custom scripts written in the MATLAB programming language. The figures presented in Chapter $\mathrm{V}$ were generated also by these tools.

\section{RESULTS AND DISCUSSION}

We discuss the results of the simulations by analyzing the effect of each of the input parameters, one after another. The main results are read from the generated figures, however, numerical data is also available as the output of the simulations. 


\section{A. The effect of time between injections $T_{s}$}

The total amount of drug required for the therapy depends on the length of the rest periods (Figs. 1-5). The function has a clear minimum point between one and four days (depending on the other parameters) for PLD6 (cf. Figs. 1, 2, and 4). This schedule is optimal because the minimal injection dose (defined by parameter $T O L$ ) is enough to be administered at each treatment during the therapy. This holds obviously also for shorter rest periods, however, shorter periods means more injections, and so, the total amount of administered drug becomes greater when the full course of the therapy is considered.

\section{B. The effect of achievable distance from the reference output $\epsilon$}

There is no reason to lower the achievable distance from the reference output below 0.01 (see Fig. 1), because much more drug is needed without having measurably better results in the final tumor volume. Allowing a greater distance, however, may lead to unsuccessful therapy, see e.g. [7].

\section{The effect of injection dose limit $U_{M A X}$}

The default upper limit of the injection dose used by our algorithm is the MTD value, which is $8 \mathrm{mg} / \mathrm{kg}$ for the applied drug, i.e., pegylated liposomal doxorubicin. Setting this limit to lower values helps to approximate the analytical minimum point more closely for rest periods shorter than two days or longer than 6 days (Fig. 2), which reduces in those cases also the total amount of required drug for the entire therapy, since this parameter directly affects the desired tumor volume.

\section{The effect of algorithm accuracy TOL}

Although running the search algorithm for more iterations may result in lower injection doses as the algorithm is enabled to approximate the analytical minimum point closer, those minimal improvements are practically irrelevant for such low doses in the scale of $\mu \mathrm{g} / \mathrm{kg}$. On the other hand, limiting the number of iterations (widening the tolerance interval) may lead to drastic increase in the amount of drug required, especially for short rest periods, where very low injection doses are sufficient to reduce the tumor volume In other words, if the tolerance is higher, the algorithm will stop very soon and propose large doses, which are far from the optimum. This phenomena can be seen in Fig. 3 .

\section{E. The effect of therapy length $L$}

Figure 4 illustrates that the optimal schedule of the injections (i.e,. the rest period which minimizes the total amount of drug) is independent of the total length of the therapy. Note that 30 days are not enough to reduce the tumor volume to zero in most of the cases. In turn, 90 days are unnecessarily long, because the tumor size will be eliminated in ca. 60 days independently of the duration of the rest periods.

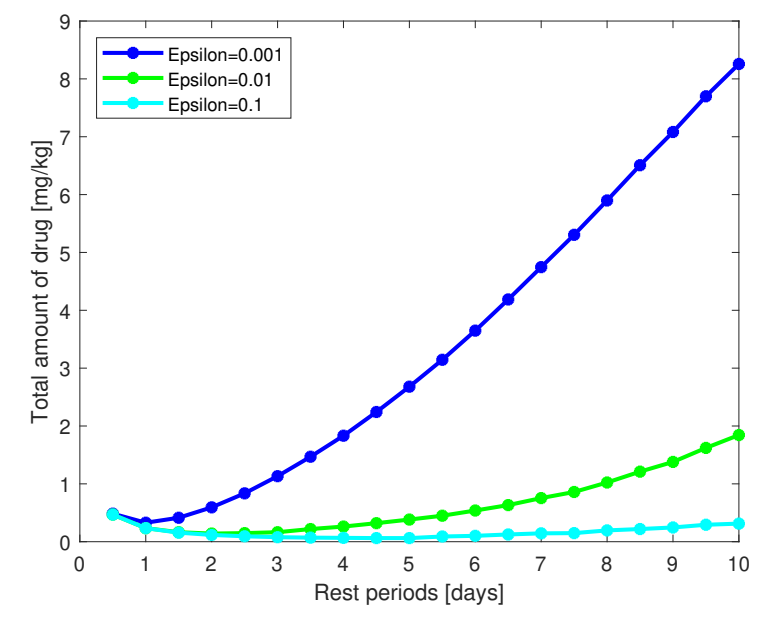

Fig. 1. The effect of achievable distance from the reference output. Patient: $P L D 6, U_{M A X}=8 \mathrm{mg} / \mathrm{kg}, T O L=8 * 10 e-3 \mathrm{mg} / \mathrm{kg}, L=60$ days

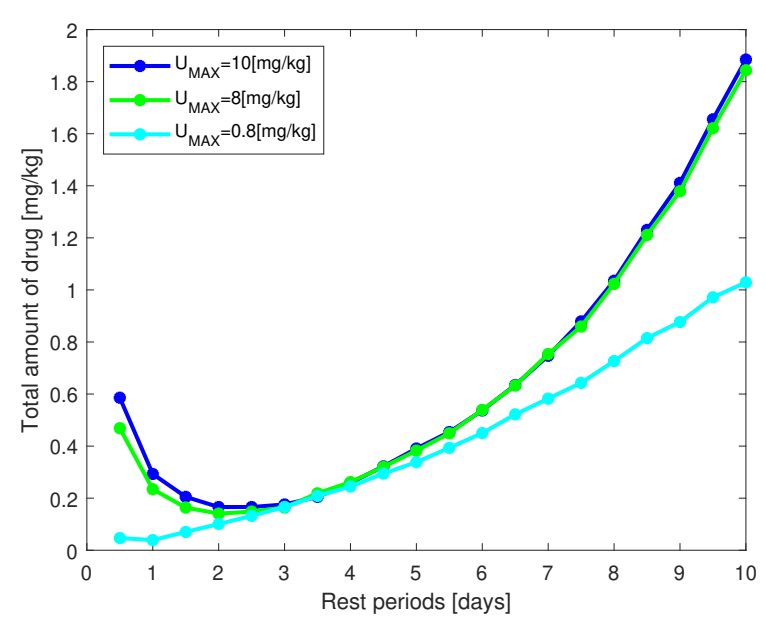

Fig. 2. The effect of injection dose limit. Patient: $P L D 6, T O L=$ $U_{M A X} / 1000 \mathrm{mg} / \mathrm{kg}, L=60$ days

\section{F. Inter-patient variability}

The minimal total amount of drug correlates to the median effective dose (ED50) parameter, which is identified individually for the patients under test. Patients with higher ED50 and clearance values (PLD2 and PLD10) need higher amounts of drug only in the cases of longer rest periods applied. Rest periods below the desirable two - four days period are not optimal, because the drug will not be depleted from the body in such a short time. This is related to the clearance parameter of the patient. The simulation results for the different patients are visualized in Fig. 5. (The parameter sets of the patients are described in Table I.)

\section{CONCLUSION}

We performed an exhaustive in-silico analysis on our therapy generation algorithm and concluded that an optimal schedule of LDM therapy can be found, and the schedule only slightly depends on the input parameter set of the ther- 


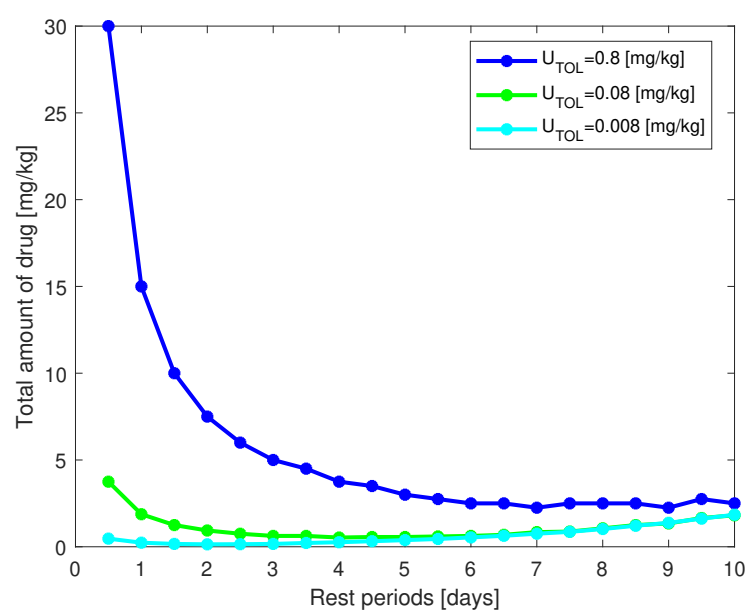

Fig. 3. The effect of algorithm accuracy. Patient: $P L D 6, U_{M A X}=$ $8 \mathrm{mg} / \mathrm{kg}, \epsilon=0.01, \mathrm{~L}=60$ days

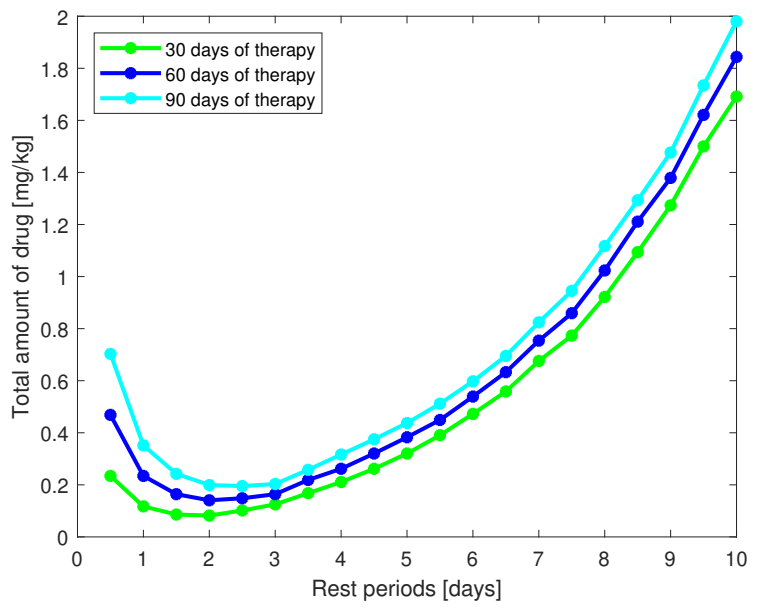

Fig. 4. The effect of therapy length, Patient: $P L D 6, U_{M A X}=8 \mathrm{mg} / \mathrm{kg}$, $\epsilon=0.01, T O L=8 * 10 e-3 \mathrm{mg} / \mathrm{kg}$

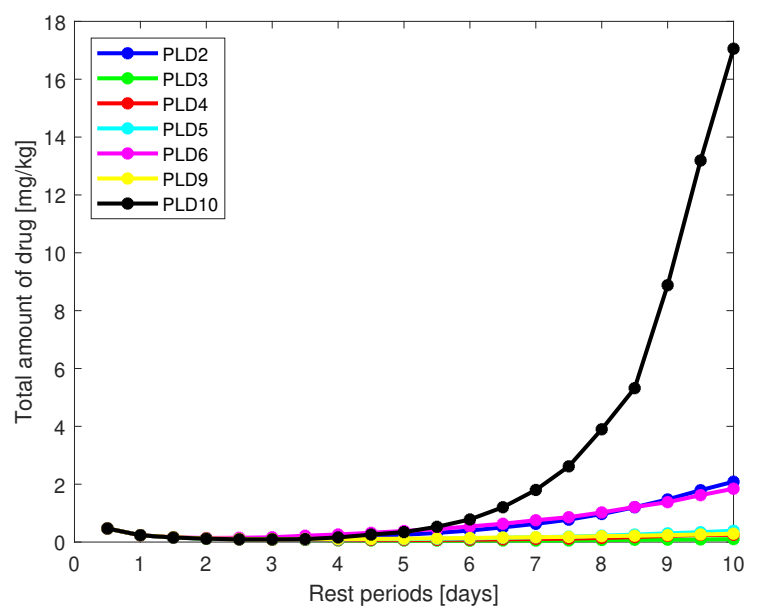

Fig. 5. Inter-patient variability. $U_{M A X}=8 \mathrm{mg} / \mathrm{kg}, \epsilon=0.01, T O L=$ $8 * 10 e-3 \mathrm{mg} / \mathrm{kg}, L=60$ days apy generation algorithm. The optimal schedule is defined by the ED50 and clearance parameters of the individual patients, which meets previous expectations. Limitation is that we performed only a quantitative analysis yet. Our future goals are to describe the correlations between the parameters and the generated therapies qualitatively, and to characterize the generated therapies with less parameters in order to simplify the therapy generation process.

\section{REFERENCES}

[1] T. Browder, C. E. Butterfield, B. M. Kraling, B. Shi, B. Marshall, M. S. O'Reilly, and J. Folkman, "Antiangiogenic scheduling of chemotherapy improves efficacy against experimental drug-resistant cancer," Cancer Research, vol. 60, pp. 1878-1886, April 2000.

[2] J. West and P. K. Newton, "Chemotherapeutic dose scheduling based on tumor growth rates provides a case for low-dose metronomic highentropy therapies," Cancer Research, vol. 77, pp. 6717-6728, October 2017.

[3] D. Loven, E. Hasnis, F. Bertolini, and Y. Shaked, "Low-dose metronomic chemotherapy: from past experience to new paradigms in the treatment of cancer," Drug Discovery Today, vol. 18, no. 3,4, pp. 193201, February 2013.

[4] N. André, M. Carré, and E. Pasquier, "Metronomics: towards personalized chemotherapy?" NATURE REVIEWS - CLINICAL ONCOLOGY, vol. 11, pp. 413-431, July 2014.

[5] T. Yang, Impulsive Control Theory. Berlin, Germany: Springer, 2001.

[6] N. A. Perestyuk, V. A. Plotnikov, A. M. Samoilenko, and N. V. Skripnik, Differential Equations with Impulse Effects. Berlin, Germany: De Gruyter, 2011.

[7] D. A. Drexler and L. Kovács, "Optimization of impulsive discretetime tumor chemotherapy," in Proceedings of the 2019 IEEE 1st International Conference on Societal Automation., 2019.

[8] D. A. Drexler, T. Ferenci, A. Fúredi, G. Szakács, and L. Kovács, "Experimental data-driven tumor modeling for chemotherapy," in Proceedings of the 21st International World Congress of the International Federation of Automatic Control, 2020, accepted for publication.

[9] J. P. L. dos Santos Belfo, "Optimal impulsive control for cancer therapy," Tecnico Lisboa, Tech. Rep., 102018.

[10] F. Cacace, V. Cusimano, A. Germani, P. Palumbo, and F. Papa, "Closed-loop control of tumor growth by means of anti-angiogenic administration," Mathematical Biosciences \& Engineering, vol. 15, no. 4, pp. 827-839, 2018. [Online]. Available: https://doi.org/10. 3934/mbe.2018037

[11] D. A. Drexler, J. Sápi, and L. Kovács, "A minimal model of tumor growth with angiogenic inhibition using bevacizumab," in Proceedings of the 2017 IEEE 15th International Symposium on Applied Machine Intelligence and Informatics, 2017, pp. 185-190.

[12] D. A. Drexler, J. Sápi, and L. Kovács, "Modeling of tumor growth incorporating the effects of necrosis and the effect of bevacizumab," Complexity, pp. 1-11, 2017.

[13] D. A. Drexler, T. Ferenci, A. Lovrics, and L. Kovács, "Modeling of tumor growth incorporating the effect of pegylated liposomal doxorubicin," in Proceedings of the IEEE 23rd International Conference on Intelligent Engineering Systems, 2019, pp. 369-374.

[14] D. A. Drexler, T. Ferenci, A. Lovrics, and L. Kovács, "Tumor dynamics modeling based on formal reaction kinetics," Acta Polytechnica Hungarica, vol. 16, pp. 31-44, 2019.

[15] A. Füredi, K. Szebényi, S. Tóth, M. Cserepes, L. Hámori, V. Nagy, E. Karai, P. Vajdovich, T. Imre, P. Szabó, D. Szüts, J. Tóvári, and G. Szakács, "Pegylated liposomal formulation of doxorubicin overcomes drug resistance in a genetically engineered mouse model of breast cancer," Journal of Controlled Release, vol. 261, pp. 287-296, 2017.

[16] D. A. Drexler, J. Sápi, and L. Kovács, "Optimal discrete time control of antiangiogenic tumor therapy," IFAC-PapersOnLine, vol. 50, no. 1, pp. $13504-13509,2017,20$ th IFAC World Congress. 
B. Péceli et al. • Optimal scheduling of low-dose metronomic chemotherapy: an in-silico analysis

000416 Authorized licensed use limited to: University of Exeter. Downloaded on July 02,2020 at 13:38:19 UTC from IEEE Xplore. Restrictions apply. 